\title{
Globe
}

Revue internationale d'études québécoises

\section{Marie-Pier Luneau : Lionel Groulx. Le mythe du berger. Montréal, Leméac, 2003}

\section{Michel Lacroix}

Volume 7, numéro 2, 2004

URI : https://id.erudit.org/iderudit/1000870ar

DOI : https://doi.org/10.7202/1000870ar

Aller au sommaire du numéro

Éditeur(s)

Globe, Revue internationale d'études québécoises

ISSN

1481-5869 (imprimé)

1923-8231 (numérique)

Découvrir la revue

Citer ce compte rendu

Lacroix, M. (2004). Compte rendu de [Marie-Pier Luneau : Lionel Groulx. Le mythe du berger. Montréal, Leméac, 2003]. Globe, 7(2), 208-212.

https://doi.org/10.7202/1000870ar d'utilisation que vous pouvez consulter en ligne.

https://apropos.erudit.org/fr/usagers/politique-dutilisation/ 
représentatif de la spécificité de la recherche au Québec, de son dynamisme, de l'exigence de rigueur et de la volonté de renouveau qui y règnent.

La qualité des contenus et l'organisation de l'ensemble en font un excellent ouvrage de formation, un outil de recherche et un ouvrage de consultation. La qualité de l'index, de la table des matières et du répertoire thématique assure en effet une consultation rapide et efficace, et le foisonnement de bibliographies permet au chercheur d'approfondir davantage les différents secteurs de la culture.

Un ouvrage si ambitieux et si rigoureux pourrait envisager, dans de futures rééditions, d'ouvrir encore certains espaces de recherche sur la culture. Ainsi, une section pourrait être consacrée à la langue dans ses rapports à la culture et à la littérature au Québec (identité, traduction, culture), ou encore à la culture québécoise sous le regard étranger (projets de recherche internationaux, développement des études québécoises à l'étranger), ce qui contribuerait à rendre visibles le pouvoir de fascination de la culture québécoise et les apports de la recherche sur celle-ci.

Carmen Mata Barreiro Universidad Autónoma de Madrid (Espagne)

\section{Marie-Pier Luneau}

Lionel Groulx. Le mythe du berger.

Montréal, Leméac, 2003.

À la bourse des valeurs intellectuelles, les écrivains, intellectuels et phénomènes sociaux de la première moitié du vingtième siècle québécois sont en forte hausse. Depuis quelques années, en effet, des chercheurs de multiples disciplines concentrent leurs regards de ce côté, modifiant considérablement ce que l'on croyait savoir sur cette période. Des ouvrages aussi divers que ceux de Louise Bienvenue, Daniel Chartier, Alain G. Gagnon et Michel Sarra-Bournet, Hélène PelletierBaillargeon, Esther Trépanier et Jean-Philippe Warren, ont déplacé les repères historiques habituels et montré, entre autres choses, que bien des traits associés à la culture et à la société québécoises modernes ne 
surgirent pas dans leur forme achevée à la grande époque de la Révolution tranquille, telle Athéna de la cuisse de Jupiter, mais furent esquissés dans les années vingt, trente ou quarante, voire plus tôt encore.

Cette convergence sans coordination, signe de courants profonds, est marquée par le renouveau d'intérêt pour l'œuvre de Lionel Groulx : pas moins de quatre ouvrages lui ont été consacrés au cours des deux dernières années, dont ceux de Gérard Bouchard et de Frédéric Boily, ainsi que le $3^{e}$ tome de l'édition critique de sa correspondance, sous la direction de Giselle Huot, Juliette Lalonde-Rémillard et Pierre Trépanier. Dans cette fournée groulxienne, la monographie de Luneau se démarque par sa perspective résolument sociolittéraire. Là où la plupart des travaux, les tout derniers comme ceux qui les précédèrent, ont interrogé Groulx comme historien et intellectuel, c'est la carrière de l'écrivain qui est ici examinée. Celle-ci, en effet, est singulière : comment le "petit paysan - au bagage culturel fort étroit, dépourvu d'appuis et désargenté, a-t-il pu marquer à ce point l'histoire littéraire et intellectuelle, pour devenir non seulement un des premiers auteurs de best-sellers mais un mythe vivant? Pour Luneau, l'exceptionnalité de ce parcours découle d'une stratégie cohérente déployée par Groulx dès ses premiers textes publiés, en 1905 et 1906, stratégie dont elle fait une démonstration extrêmement convaincante au fil d'un essai vivant, parfois même primesautier, à des lieues de la prose empesée si courante dans les ouvrages écrits à l'ombre de l'université (Le Mythe du berger origine d'une thèse de doctorat déposée à l'Université de Sherbrooke en 2001).

Pour analyser la trajectoire d'écrivain de Groulx, elle reprend l'opposition développée par Alain Viala, dans Naissance de l'écrivain, entre stratégie de la réussite et stratégie du succès, la première étant le fait d'écrivains qui misent sur les institutions, tentent de cumuler les positions et visent à obtenir un à un, patiemment, des acquis solides, alors que la seconde caractérise ceux qui cherchent d'abord à conquérir le public, en visant des gains rapides, réalisés avec éclat, dans l'espoir de reconvertir plus tard la renommée publique en reconnaissance littéraire (capital symbolique). Originellement conçue pour décrire le xvile siècle français, l'approche de Viala colle d'autant plus à l'univers canadien-français du début du $\mathrm{XX}^{\mathrm{e}}$ siècle que, comme au xvie siècle, on peut y voir la littérature commencer à constituer un espace social distinct. Son application au cas de Lionel Groulx emporte absolument l'adhésion. Se basant sur une lecture minutieuse des Mémoires et de la gigantesque 
correspondance de ce dernier, Luneau nous montre un écrivain faisant constamment preuve d'audace (stratégiquement parlant), prêt à défier ses supérieurs ou les hommes en place, quitte à s'opposer à ses anciens alliés ; un écrivain à ce point soucieux d'assurer une large diffusion à ses articles, brochures, monographies et romans qu'il mit sur pied un vaste réseau de "propagande ", essentiellement clérical, qui assurait les achats dans les collèges aussi bien que des campagnes de critiques favorables; un écrivain, enfin, hanté par la nécessité de "forcer "les portes du succès, quitte à publier trop vite, à susciter des envies, à écrire lui-même, sous pseudonyme, des notes sur ses œuvres; un écrivain de combat, en un mot, qui plaçait la victoire, immédiate si possible, au-dessus de tout et ne reculait devant aucun moyen pour l'obtenir.

Cette stratégie du succès, présente dès les tout débuts, lorsque, professeur au Séminaire de Valleyfield, il passe outre à la timidité de ses supérieurs ou aux réticences de ses amis pour publier ses premiers textes, se manifeste avec éclat au moment de la publication de L'Appel de la race, en 1922. Sous pseudonyme, Groulx annonce et louange le roman des mois avant sa parution, sollicite des comptes rendus à de multiples connaissances et oppose la faveur du peuple (1 200 exemplaires sont vendus la première semaine) aux reproches des pédants (parmi lesquels il place aussi bien René du Roure que Camille Roy). On voit alors, selon Luneau, l'auteur des Rappaillages afficher " son indépendance presque provocatrice à l'égard des autres agents du champ * (p. 102-103), et développer un double discours qui camoufle sa très grande implication dans la défense de l'œuvre sous une image d'auteur planant sereinement au-dessus des mesquineries polémiques.

Cette démonstration modifie radicalement l'image de l'abbé, sacralisée et institutionnalisée, que l'on aurait à première vue associée à la stratégie de la réussite. Dans certains cas, cependant, on peut se demander s'il n'y a pas eu, chez Groulx, poursuite d'une double stratégie, étant donné les velléités épisodiques mais récurrentes d'obtenir l'estime et l'appui de certaines autorités, françaises en particulier. N'a-t-il pas malgré tout choisi de faire partie de la Société Royale du Canada, institution officielle s'il en est? Mais cela ne remet pas en question la prédominance très nette de la stratégie du succès. Qu'un auteur ait désormais pu choisir cette dernière pourrait par ailleurs être vu comme un indice majeur mais négligé de l'établissement d'un champ littéraire solide au Québec, d'un "premier champ", comme le dirait Viala. Sur ce plan, l'ouvrage de 
Luneau contribuera aussi à approfondir l'étude de la vie littéraire québécoise de la période; on peut d'ailleurs espérer que d'autres études de trajectoires compléteront ce portrait et fourniront des points de comparaison. Ceci sera particulièrement utile en ce qui concerne les éditions à compte d'auteur, qui obligeaient les écrivains à s'occuper tant bien que mal de leur propre diffusion, les poussant ainsi insensiblement du côté de la stratégie du succès.

Les liens entre stratégie littéraire et image de l'écrivain sont au centre de l'étude de Luneau et constituent un de ses aspects les plus intéressants. Elle a su de fort belle façon montrer qu'un parcours littéraire est tout à la fois fonction de phénomènes concrets (ventes d'ouvrages, contrats, relations entre acteurs, etc.) et fonction de la représentation qu'on s'en fait. Or, ce dernier aspect a tendance à être grandement négligé dans les études sur les trajectoires et les sociabilités littéraires. On voit ainsi apparaître peu à peu, dès 1912, la figure du berger, celle du guide de la jeunesse tout d'abord, bien rapidement transformée, lors du passage de Valleyfield à Montréal, en celle de maître intellectuel de la nation tout entière, image relayee par de jeunes disciples enthousiastes. Les années vingt constituent à cet égard celles de l'apogée de l'influence de Groulx, dans la lecture de Luneau. Les décennies suivantes marqueraient en effet la perte d'influence de l'abbé auprès des jeunes générations, malgré ses ventes toujours aussi honorables, puis la solitude croissante de celui-ci quand, après la guerre, même le public ne le suit plus. Très solide, dans les deux premiers chapitres, cette reconstitution semble cependant un peu plus rapide dans les suivants, un peu trop centrée sur les relations de l'écrivain avec les éditeurs et pas assez sur la place de Groulx dans le champ intellectuel québécois. Certes, la décision de ne pas aborder ses idées explique ce choix. Néanmoins, il accélère en quelque sorte la chute du mythe groulxien, puisque dans les années trente, plusieurs groupes de jeunes se réclament encore de ses idées à défaut de prolonger ses choix esthétiques, et ce aussi bien chez les Jeunes Canada que dans La Relève. La perspective empruntée dans Le Mythe du berger peut aussi soulever une autre critique, celle de solliciter plus souvent les mémoires que les lettres ou les discours contemporains, ce qui donne un peu l'impression que la vérité ultime de la représentation que Groulx fait de sa carrière se trouve dans les mémoires et porte à croire qu'il n'en a jamais divergé tout au long de sa vie, quels que soient les moments ou les correspondants. Peut-être cela a-t-il été effectivement le cas, seulement les preuves manquent sur ce point. 
Enfin, et sur un tout autre plan, cet ouvrage fait surgir une interrogation plus vaste quant aux rapports entre les champs : n'y a-t-il pas, à l'époque de Groulx, différenciation de plus en plus nette entre littérature et histoire? Groulx ne serait-il pas le dernier écrivain-historien du Québec, le dernier à unir dans un parcours cohérent les deux " métiers " ? Quelle que soit la réponse, cela amène à considérer les différences éventuelles quant au statut dans le champ, quant au rôle de maître. On peut par exemple se demander si la stratégie du succès est encore possible dans le domaine historique, après Groulx, l'emprise universitaire sur le champ historique limitant dès lors les itinéraires à ceux de la réussite ? Ce n'est d'ailleurs pas un des moindres mérites de cette belle étude que de lever toute une série de lièvres, que d'autres chercheurs prendront plaisir à chasser à leur tour.

Michel Lacroix

Université du Québec à Trois-Rivières

\section{Claude Gauvreau}

Lettres à Paul-Émile Borduas.

Édition critique de Gilles Lapointe, Montréal, Presses de l'Université de Montréal, coll. "Bibliothèque du Nouveau Monde ", 2002.

De nombreuses éditions critiques de qualité sur la littérature québécoise ont été publiées ces dernières années pour le plus grand bénéfice des chercheurs québécois et étrangers, qui y (re)découvrent des corpus souvent inédits, à la lumière de mises en contexte précises qui jettent un jour nouveau sur les œuvres. Les Lettres à Paul-Émile Borduas du poète automatiste Claude Gauvreau, publiées dans la prestigieuse collection Bibliothèque du Nouveau Monde des Presses de l'Université de Montréal, ne font pas exception à la règle. Gilles Lapointe, qui nous avait déjà donné de substantielles éditions critiques, récidive ainsi avec la publication des lettres enfiévrées de Gauvreau au peintre Paul-Émile Borduas, rédigées entre 1948 et 1959 , c'est-à-dire de Refus global jusqu'à la mort de Borduas. Le lecteur y trouvera en outre une introduction extrêmement complète de Lapointe, accompagnée d'une chronologie 